\title{
¿Trabajo Barato?: Tiempo, Capital y la Reproducción de la Naturaleza Humana
}

\section{JASON W. MOORE*}

Por esta política de desperdicio y destrucción de las fuerzas productivas del hombre, el capitalismo se condena a sí mismo... Privado del consumo histórico del valor del trabajo producido fuera de su esfera y del principal freno a la caída de tasas de beneficio, el capitalismo bien podría haberse mostrado como un modo

de producción para movilizar con éxito, como lo hizo al principio, las fuerzas productivas, y por lo tanto garantizar el progreso. La llegada de la crisis "final" estaría entonces presagiada por la situación presente. (Meillasoux, 1981).

a dialéctica de la capitalización y de la apropiación se basa, fundamentalmente, en las relaciones a través de las cuáles los humanos son re/producidos. Así como la centralidad de la Fuerza de Trabajo Barata. Sin él, la acumulación quiebra. Para Marx,

"La reproducción de la fuerza de trabajo, que debe ser incesantemente reincorporada al capital como su medio de valorización [La propia expansión del capital], que no puede liberarse del capital, y cuya esclavitud al capital es sólo encubierta por la variedad de individuos capitalistas a los que se vende, forma de hecho, un [esencial] factor en la reproducción del propio capital. La acumulación del capital es así la multiplicación del proletariado ${ }^{1 . "}$

A la famosa observación de Marx, nosotros debemos añadir ahora: la acumulación del capital es la multiplicación del proletariado, es la apropiación del trabajo/energía no pagada. Convirtiendo la "sangre en el capital"2, la relación del capital despliega poco a poco la producción de riqueza como un valor, y la apropiación del trabajo no remunerado (la re/producción de vida) como la condición de dicho valor. El capitalismo hace esto bajo condiciones geográficas específicas. El régimen del trabajo social abstracto emerge -incluso antes que la industria a gran escala- bajo condiciones de rápida expansión geográfica ${ }^{3}$. Pero las implicaciones de esta relación profundizan más que los argumentos acerca de la expansión global

\footnotetext{
1 MARX, Karl, Capital, Vol. I, 1977, pp. 763-764; Palabras entre corchetes de MARX, Karl, Capital, Vol. I, 1967, pp. 575-576. Añadido el énfasis por el autor.

2 Ibídem p. 382.

3 MOORE, Jason, Ecology and the Rise of Capitalism, 2007; TILLY, C., Demographic Origins of the European Proletariat, CRSO Center for Research on Social Organization, University of Michigan, Working Paper No 207, 1979.
}

\section{*Jason W. MOORE, historiador medioambiental y economista político en el Departamento de Sociología en la Universidad de Binghamton y coordinador de la World Ecology Research Network.}

Este artículo ha sido traducido con el permiso de la editorial, así como de su autor Jason W. Moore. Publicado originalmente como MOORE, Jason W., Capitalism in the Web of Life, Editorial Verso, Londres, 2015. Capítulo 9, pp. 221-240.

Traducción: Gonzalo VITÓN doctorando con contrato FPUMECD en el Departamento de Historia Contemporánea de la Universidad Autónoma de Madrid.

DOI: $10.15366 /$ relacionesinternacio nales2017.36.011 
como pivote del crecimiento del capitalismo ${ }^{4}$. El trabajo social abstracto no crea fronteras al ser un proceso-frontera en sí mismo. Esa frontera es el límite entre una vida mercantilizada o no mercantilizada, y el capital se mueve a lo largo de ese límite a través del mapeo y la cuantificación de técnicas de la naturaleza social abstracta. Para todos los personajes "autónomos" de El Capital, la producción de excedentes no es solo la proletarización del trabajo y la acumulación de capital, sino la producción de espacios globales de apropiación.

Marx ha sido frecuentemente criticado por reducir la reproducción de la fuerza de trabajo al consumo de bienes. Se nos ha dicho que en el proceso ha ignorado las contribuciones del trabajo no remunerado (especialmente el trabajo doméstico) ${ }^{5}$. Esto no parece ser del todo correcto. En su clásico discurso de "El Día del Trabajo", Marx deja claro que cualquier contención de la reproducción de la fuerza de trabajo dentro del sistema de bienes, llevaría rápidamente a un incremento de los costos laborales y a una acumulación tambaleante. "Parecería que el interés del propio capital apunta en la dirección de un día normal de trabajo"6. Cuanto más largo el día de trabajo, y cuánto más intenso fuera este, mayor es el "deterioro de la fuerza de trabajo humana"7. El capitalismo no es por nada -incluso antes de la era de la industria a gran escala- "el primer sistema... que provee... de ímpetus a la patología industrial"8. A primera vista, dicho deterioro de la fuerza de trabajo parece ir en dirección contraria al propio interés del capital, ya que la fuerza de trabajo "agotada" debe ser remplazada, lo que es una propuesta "cara". Pero mientras el "valor de la fuerza de trabajo incluye el valor de los bienes necesarios para la reproducción del trabajador", el valor de esos bienes está determinado por una combinación del trabajo -remunerado y no remunerado- capitalizado y apropiado. Valorizar directamente la fuerza de trabajo, determina el valor de los bienes necesarios para la reproducción del trabajador. Al mismo tiempo, el trabajo de reproducción no remunerado codetermina el tiempo de trabajo socialmente necesario que establece el valor de esos bienes. Como hemos visto, este trabajo/energía no remunerada no está limitado al ámbito del hogar, sino que se extiende al completo sistema de la Naturaleza Barata. El tiempo de trabajo necesario es coproducido a través de la capitalización y apropiación.

¿Por qué coproducido? Porque el capital necesariamente se perfila en zonas de trabajo no mercantilizado (trabajo no remunerado); la reproducción de la fuerza de trabajo ocurre solo parcialmente dentro de la zona de producción e intercambio mercantilizado. Sostener todos los costes de la reproducción de los hogares dentro del sistema de bienes llevaría rápidamente al proceso de acumulación a una parada. Los hogares totalmente proletarios son por lo tanto bastante extraños en el capitalismo incluso hoy, limitados prácticamente en su totalidad a trabajadores profesionales bien pagados (abogados, doctores, profesores, etc.). Históricamente, incluso en el núcleo de la proletarización, la reproducción de la fuerza de trabajo ha dependido en todas sus formas del trabajo no remunerado, o trabajo remunerado a un nivel insuficiente para reproducir por sí solo la fuerza de trabajo. El último punto

${ }^{4}$ Cf. WALLERSTEIN, The Modern World-System I, 1974

5 MEILlASSOUX, C. Maidens, Meal and Money, Cambridge University Press, Cambridge, 1981; FEDERICI, S, Revolution at Point Zero, PM Press, Oakland, 2012.

6 MARX, Karl, Capital... op. cit., p. 377. Énfasis añadido por el autor.

7 Ibíd., p. 376.

8 Ibíd., p. 484.

9 Ibíd., p. 377. 
es importante, porque estamos tratando con grados relativos de trabajo no remunerado, cambiando las configuraciones del trabajo remunerado y no remunerado en el "hogar semiproletario"10. Por ejemplo, en Norteamérica a principios del siglo XX la mitad de todas las mujeres inmigrantes en ciudades americanas -en un momento en que la mayoría de los inmigrantes estaban en las grandes ciudades- trabajaban como internas remuneradas, una actividad que incluía todos los tipos de limpieza, cocina y trabajo emocional. De hecho, incluso en pueblos industriales de tamaño medio -Como Muncie, Indiana- cerca de la mitad de todas las familias de clase trabajadora cultivaban pequeños jardines de verduras en la década de los años $20^{11}$.

Hay una tentación de aceptar esta realidad del trabajo social abstracto como coproducida a través de la capitalización y apropiación, y al mismo tiempo negar que Marx reconoció el problema ${ }^{12}$. Y si fuera una cuestión simplemente de si Marx estaba en lo cierto -o equivocado- difícilmente merecería la pena ser puntilloso con ello. Haríamos bien en asistir a cómo Marx construyó el argumento acerca de la reproducción de la fuerza de trabajo. Pues Marx se mueve constantemente de abstracciones generales, como la producción, la población y el intercambio en general, hacia abstracciones sucesivamente más específicas o determinadas ${ }^{13}$. En un texto iluminador, Marx ofrece tanto una abstracción general como determinada del trabajo, moviéndose de lo anterior a lo último:

"Como una actividad útil dirigida a la apropiación de factores naturales en una forma u otra, el trabajo es una condición natural de la existencia humana, la condición del intercambio material entre hombre y naturaleza, bastante independiente de la forma de la sociedad. Por otro lado, el trabajo que plantea el valor-cambio [mercantilización de la fuerza de trabajo] es una forma social específica de trabajo ${ }^{14}$." $^{\prime}$

En El Capital, encontramos a Marx moviéndose consistentemente de un modelo "puro" de acumulación de capital hacia abstracciones más determinadas. El argumento en "El Día del Trabajo" ofrece una teoría implícita de la tendencia del capitalismo hacia la producción insuficiente de la fuerza de trabajo y de los mecanismos que no son del mercado para atenuar esta contradicción. Esto es especialmente evidente en su tratamiento de la reproducción de la fuerza de trabajo. La abstracción inicial de Marx del valor de la fuerza de trabajo definida por el valor de los bienes, es posteriormente modificada por una abstracción nueva e históricamente determinada donde la zona de apropiación es central15. Aquí, las capas "latentes" del ejército reserva del trabajo son cruciales ${ }^{16}$. Habiendo "dimensionado las fuerzas vitales de la gente en sus raíces más profundas... la degeneración de la población industrial

${ }^{10}$ SMITH, J., y WALLERSTEIN, I., Creating and Transforming Households, Cambridge University Press, Cambridge, 1992.

${ }^{11}$ GORDON, L., "US Women's History", en FONER, E. (ed.), The New American History, Temple University Press, Philadelphia, 1990, p. 271.

12 Cf. FEDERICI, S., Revolution at Point Zero, PM Press, Oakland, 2012.

13 MARX, Karl, Grundrisse (1973); MURRAY, P., Marx's Theory of Scientific Knowledge, NJ: Humanities Press, Atlantic Highlands, 1988.

${ }^{14}$ MARX, K., A Contribution to the Critique of Political Economy, traducido por Charles H. Kerr \& Co., N.I. Stone, Chicago, 1904 [orig. 1859], p. 33.

15 MARX, Karl, Capital..., op. cit., pp. 276-277.

${ }^{16}$ Ibíd., p. 796. 
está solo retrasada por la constante absorción de primitivos y naturales [seres humanos 'físicamente incorruptos'] elementos del campo, ${ }^{117}$ un movimiento más tarde examinado por Marx en su famosa disertación de la acumulación primitiva. Citando a Cairnes con su visto bueno, Marx observa que si la fuerza de trabajo puede ser:

"Suministrada por dominios extranjeros... la duración de la vida [del trabajador] se convierte en una cuestión menor mientras su productividad dure. Es por consiguiente la máxima del negocio esclavista en países importadores de esclavos, que la economía más efectiva es la que saca provecho del esclavo en el mínimo espacio temporal con el máximo esfuerzo que es capaz de sacar adelante ${ }^{18} . "$

A lo que Marx añade: "Mutato nomine te fabula narratur [El nombre cambia, pero el cuento es el mismo]. Para el comercio de esclavos, véase el mercado de trabajo, para Kentucky y Virginia [en el comercio de esclavos], Irlanda y los distritos agrícolas de Inglaterra, Escocia y Gales, para África, Alemania ${ }^{19}$. Para la fuerza de trabajo, véase la naturaleza. Marx hace la conexión directamente:

El capital no pregunta acerca de la duración de la vida de la fuerza de trabajo. Lo que interesa es simple y puramente la máxima fuerza de trabajo que pueda ponerse en marcha en un día de trabajo. Consigue este objetivo al acortar la vida de la fuerza de trabajo, de la misma forma que los granjeros avariciosos arrancan más producción del suelo robándole su fertilidad ${ }^{20}$.

De la misma forma... He ahí una reveladora declaración acerca de cómo la relación del capital se desarrolla poco a poco a través del oikeios. Como vimos en el capítulo tercero ${ }^{21}$, el "proceso interdependiente del metabolismo social" se convierte en un singular -pero históricamente diferenciado- metabolismo de la naturaleza humana y extrahumana. Aquí, podemos iluminar la simbólica violencia del binarismo Cartesiano, oscureciendo los tejidos conectivos entre la "reducción de la vida" del trabajador, y el "robo" del suelo.

Es difícil ver esos tejidos conectivos en la mayoría del Pensamiento Verde. Despierta a cualquier medioambientalista a mitad de la noche y pregúntale: "¿Dónde vemos el agotamiento y la merma?" La respuesta está preparada: en la flora y fauna, en el suelo y los recursos. Pero ¿qué ocurre si invertimos la respuesta, y empezamos por el punto de vista del agotamiento del trabajador, y del agotamiento de los sistemas de trabajo? Dicha inversión no necesita ser antropocéntrica; a través de ella, puede que iluminemos las relaciones unificadoras agotando las naturalezas humanas y extrahumanas en la ecología-mundo capitalista.

Si el agotamiento del trabajador es primordial, deberíamos hacernos una pregunta crucial: ¿Quién es el trabajador? No solo el trabajador asalariado, para estar seguros, sino todas las actividades de la vida que "trabajan" en las relaciones y valores del capitalismo. Como

\footnotetext{
${ }^{17}$ Ibíd., p. 380.

${ }^{18}$ CAIRNES, J., The Slave Power, Parker, Son and Bourn, London, 1862; MARX, Karl, Capital..., op. cit. p. 377.

19 MARX, K., Capital..., p. 376. Énfasis añadido por el autor.

${ }^{20}$ Ibíd., p. 378. Énfasis añadido por el autor.

${ }^{21}$ N.d.T.: Capítulo tercero del libro MOORE, Jason, Capitalism in the Web of Life, Editorial Verso, Londres, 2015.
} 
hemos visto, parte de este trabajo es formal, pero mucho de él no lo es. Una pequeña parte de esto ocurre en fábricas, oficinas y tiendas, pero una gran parte no lo hace. Tenemos que revisar nuestras dos mayores formas de agotamientos - "exprimir" y "aniquilar"- la primera encontrada en el Capítulo Cinco ${ }^{22}$. Más típica es la anterior: una población trabajadora dada es exprimida cuando no puede distribuir una creciente oleada de trabajo/energía en -o en apoyo de- el circuito del capital. Hoy la clase americana trabajadora no está exhausta en el sentido de inminente ruptura física; está exhausta en su capacidad de distribuir un creciente volumen de trabajo no remunerado al capital. Su potencial de distribución del trabajo no remunerado está exprimido. La proliferación de "turnos" -un segundo y un tercer turno en el trabajo remunerado y no remunerado- y la extensión neoliberal de la semana de trabajo, nos da la razón al pensar que los trabajadores americanos no pueden trabajar mucho más, o mucho más duro ${ }^{23}$. (En los márgenes, quizás, pero no mucho más que esto).

Tal agotamiento también implica nuestro segundo momento "exprimido". Este es el espectro de un absoluto -no solo relativo- descenso en los flujos de trabajo/energía. Puede verse en la subida repentina y acelerada de problemas mentales a lo largo del Norte Global desde la década de los $80^{24}$ y una epidemia de cáncer incluso después de un mayor descenso de fumadores, y ello fuera de proporción con los avances diagnósticos ${ }^{25}$. Lo que esto significa está claro: el agotamiento toma diferentes formas y no puede ser reducido a una ruptura biofísica. Más allá de los cada vez mayores problemas de salud, uno también puede fijarse en el descenso de la fertilidad -una "huelga de bebés" de las mujeres proletarias a lo largo del Atlántico Norte en las décadas recientes-, y que ahora se extiende a la industrializada Asia del Este ${ }^{26}$. Esto sugiere que, por encima de un ciclo de acumulación, las relaciones de reproducción, que una vez estuvieron fuera del nexo monetario, se han progresivamente monetizado. La reproducción se canaliza a través de las relaciones de bienes, y la parte -pero no necesariamente la masa- de trabajo no remunerado desciende o se estanca. La naturaleza humana se capitaliza cada vez más en los viejos centros de producción. La capitalización es difícil sin su política de clase: la lucha por encima de términos de reproducción y fuerza de trabajo asume cada vez más importancia. El capital es cada vez más dependiente de la mercantilización de la reproducción de la vida, más que de la no mercantilización.

Una vez más vemos la tendencia del excedente ecológico caer. Su más obvio indicador es la subida del precio de los Cuatro Grandes insumos. Trabajo, comida, energía y materias primas son cada vez más y más $\operatorname{caros}^{27}$. Los Four Cheaps han dejado de ser baratos. Normalmente esto no ocurre de repente, aunque es lo que hemos visto desde 2003. El punto en el que los Four Cheaps dejaron de ser baratos y comenzaron a encarecerse es la señal de crisis de una fase del capitalismo: dichas crisis "señalan" el agotamiento de un régimen de acumulación. Para el capitalismo neoliberal, esta señal de crisis -mucho más importante

\footnotetext{
${ }^{22}$ N.d.T.: Capítulo quinto del libro MOORE, Jason, Capitalism in the Web of Life, Editorial Verso, Londres, 2015.

${ }^{23}$ Cf. HOCHSCHILD, The Second Shift, 1989

${ }^{24}$ HHS [U.S. Department of Health and Human Services], Health United States 2010, U.S. Government Printing Office, Washington D.C., 2010.

${ }^{25}$ DAVIS, M., Planet of Slums, ed. Verso, London, 2006.

${ }^{26}$ LIVINGSTON, G., y COHN, D., "The New Demography of American Motherhood," (Pew Research, 2010), www. pewsocialtrends; economist, "Women in South Korea: A Pram Too Far," Economist, (October 26, 2013).

${ }^{27}$ Por supuesto, hay siempre desigualdades entre cada insumo, y siempre hay una variación geográfica.
} 
que el cuasicolapso del sistema financiero en 2008- comenzó alrededor del 2003. Desde entonces, el excedente ecológico ha ido cayendo, con pocos signos de un inminente retorno. Las mayores fronteras de bienes han sido ya agotadas, mientras que la masa del capital continúa creciendo.

La resolución cíclica de dichas crisis de sobreacumulación -crisis definida por la creciente masa de capital "excedente" que no puede ser reinvertido provechosamente- ha dependido de la restauración cíclica de los Four Cheaps. La caída del excedente ecológico está por lo tanto muy ligada a la contracción de oportunidades beneficiosas para la inversión en la economía real (M-C-M'). Petróleo Barato, Trabajo Barato o Metales Baratos, hacen posible nuevas innovaciones -como el ferrocarril y la máquina de vapor, o el automóvil en sus respectivas eras-. (El proceso es por supuesto en cascada, y no es un proceso lineal de primero Naturaleza Barata, después innovación histórica). Los sistemas de producción, espacios urbanos, y desarrollo de infraestructuras implícitas por estas nuevas máquinas, absorben volúmenes gigantescos de excedente de capital. De hecho, la extraordinaria historia de las sucesivas industrializaciones en el Atlántico Norte entre 1790 y 1960 -abarcando la primera, segunda y la revolución industrial fordista- puede ser contada a través de estas invenciones de época (carbón/vapor, coche/petróleo), revisa el oikeios global, y permite una creciente contribución del trabajo/energía no remunerada. Enigmáticamente, la "revolución" de la información tecnológica de los últimos 40 años se ha mostrado manifiestamente inadecuada en distribuir nuevas oleadas de trabajo/energía, absorbiendo el capital excedente, o promoviendo la productividad laboral28. Haciendo posible esas grandes olas de la industrialización, los Four Cheaps son centrales en la resolución de las repetidas crisis de sobreacumulación en el capitalismo histórico. Consecuentemente, el cíclico "fin" de los Four Cheaps, en los sucesivos ciclos de acumulación, corresponde a una creciente masa de excedente de capital sin lugar a dónde ir. El agotamiento de las fronteras de los bienes -y el crecimiento ralentizado del amplio sistema de trabajo no remunerado- está consecuente y fuertemente ligado a las formas peculiares de financiamiento que han emergido desde la década de los 70. Como la acumulación en la economía real se tambalea, una creciente parte del capital gravita en torno a las finanzas más que en torno a actividades productivas $\left(M-M^{\prime} \text { en vez de } M-C-M^{\prime}\right)^{29}$. Por supuesto, en algún punto en el futuro estas apuestas financiarizadas han de ser pagadas -o el jugador quebrará-.

El excedente ecológico -el descenso relativo de la contribución del trabajo no remunerado a la acumulación de capital- puede descender por varias razones. Entre ellas, cinco son especialmente destacadas. Una es que el nexo monetario, bajo condiciones de territorialismo moderno y de la extensión de la naturaleza social abstracta, tiende a desorganizar los acuerdos precapitalistas de poder y producción. A veces, esta desorganización llega en forma de colonialismo, como cuando la reestructuración colonial española del Perú del siglo XVII desorganizó la vida de las ciudades ${ }^{30}$. Dichas desorganizaciones son un fenómeno cíclico de la ecología-mundo capitalista. Todavía están en juego tres siglos después, como el

\footnotetext{
${ }^{28}$ FOSTER y MCCHESNEY, The Endless Crisis, 2012; GORDON, "Is US Economic Growth Over?" (2012).

${ }^{29} \mathrm{M}-\mathrm{M}^{\prime}$ entra en juego durante las sucesivas expansiones financieras del capitalismo (ver Capítulo Seis). Véase ARRIGHI, The Long Twentieth Century (1994); LEYSHON, A., y THRIFT, N., "The Capitalization of Almost Everything" en Theory, Culture and Society, vol. 24, n 7-8, 2007, pp. 97-115.

30 MOORE, "Amsterdam Is Standing on Noway, Part I" (2010)
} 
desarrollo capitalista en el África colonial que indujo a un cambio en la migración de "rotativa" a "irreversible" 31 . La propia lucha de clase es un segundo vector de la caída del excedente ecológico. Las clases trabajadoras han tendido a demandar un "salario familiar" -junto con demandas fundamentalmente para socializar especialmente los costes de producción, en atención sanitaria, pensiones de jubilados, y educación ${ }^{32}-$. Esto tuvo el efecto de "encerrar" el capital en costes de reproducción más altos, especialmente en el Norte Global desde la década de los $70^{33}$. (La emigración Sur-Norte ha sido una poderosa verificación de esta tendencia). Desde entonces, hemos visto también el crecimiento de movimientos medioambientales a lo largo del mundo -nuestro tercer vector-. Estos movimientos han empujado a los estados a limitar la contaminación, y hacerse cargo de los costes de limpieza generados por la contaminación anterior. Este es el más débil de nuestros vectores hasta el momento, porque ha sido posible -hasta ahora- aplazar los costes en el tiempo, a la siguiente generación, y de desplazar los costes en el espacio, del Norte al Sur. El vector de incremento de costes es posiblemente el más fuerte en las décadas venideras, una cuestión que exploraremos en el siguiente capítulo ${ }^{34}$. Un cuarto factor es la tendencia a estrategias de simplificación radical, como el monocultivo, quitar nutrientes de los agro-ecosistemas, y producir un ambiente favorable a plagas y malezas. Esto tiende a un incremento de insumos tóxicos y de energía, los cuáles propiamente tienen un coste cada vez más alto. Finalmente, la caída del excedente ecológico implica también la disminución de las fuentes de energía y minerales, las cuáles como en la agricultura, tienden a atraer insumos cada vez más costosos -y tóxicos- (por ejemplo, minería de oro con cianuro, fracturación hidráulica, excavaciones petroleras en mar abierto).

Si la contribución relativa decreciente de trabajo no remunerado es tal problema, ¿Por qué el capital la tolera entonces, y a veces estimula fuertemente la capitalización de la reproducción? A fin de cuentas, el capital lo hace por dos grandes razones. Primera, traer los procesos de reproducción al circuito del capital, permite a las agencias capitalistas particulares (empresas) tener ganancias a corto plazo en la competitiva lucha por compartir la plusvalía mundial. Una empresa necesita suministro de fuerza de trabajo de forma regular y que no sea inferior al de materias primas. Segundo, en un nivel sistémico, la mercantilización de la fuerza de trabajo, especialmente durante periodos de estancamiento, incrementa el consumo de bienes ${ }^{35}$. La mercantilización de los alimentos es obviamente central en este punto, y la experiencia neoliberal de "subconsumo forzado" (hambre) apenas se opone a la mercantilización de los alimentos.

La fuerza de trabajo es instructiva, pues nos desafía a pensar a través de las unidades diferenciadas del capitalismo-en-la-naturaleza. El capitalismo, como indica Marx, agota el suelo y al trabajador a través de una singular, por no decir desigual, relación. Si bien dicho

\footnotetext{
31 MEILLASSOUX, Maidens, Meal and Money, 1981, p. 110.

32 WALLERSTEIN, Historical Capitalism, 1983.

33 SMITH, J., "Transforming Household" en Social Problems, vol. 34, no 5, 1987, pp. 416-436.

${ }^{34}$ N.d.T.: Capítulo décimo del libro MOORE, Jason, Capitalism in the Web of Life, Editorial Verso, Londres, 2015.

35 "Una de las principales fuerzas tras la proletarización han sido las propias fuerzas de trabajo mundiales. Ellas han entendido, normalmente mejor que sus autoproclamados portavoces intelectuales, como de mayor es la explotación [yo diría apropiación] en los hogares semi-proletarios que en los hogares más proletarizados" (WALLERSTEIN, Historical Capitalism, 1983, pp. 36-37.
} 
agotamiento es absurdo, no es irracional. A mediados de siglo, la capitalización de los costos de reproducción tiende a maximizar el trabajo no remunerado. Pasado el medio siglo, la parte relativa del trabajo no remunerado comienza a estancarse y después a descender. La composición capitalizada de la naturaleza crece. Los excedentes ecológicos caen. Como resultado de ello hay dos consecuencias: Los costos de la reproducción para el capital se incrementan, y el capital fluye hacia nuevas fronteras laborales. ( $Y$ a menudo -esta es la historia del capitalismo norteamericano especialmente- el trabajo se ha movido hacia los centros capitalistas dinámicos). Mientras parecería que el propio interés del capital señala en la dirección de regímenes de reproducción "sostenible", el corto plazo del capital y la flexibilidad de la reproducción socio-ecológica propulsa secuencias serializadas de expansión/ depresión en la historia del capitalismo -fundamentado en las tendencias exhaustivas de capitalización y apropiación-. Estas contradicciones son atenuadas a través de los ritmos temporales distintivos de la producción de bienes y la reproducción socio-ecológica. Mientras el tiempo del trabajo remunerado es "lineal y orientado al tiempo", el trabajo no remunerado de la reproducción del hogar "se fundamenta en ritmos recurrentes y patrones de actividades que son a menudo cíclicos más que lineales, están basados en las tareas en vez de en el tiempo, e incrustados en significado"36. El capital se aprovecha de la flexibilidad del trabajo reproductivo -hasta cierto punto, puede ser moldeado alrededor de las disciplinas de tiempo abstracto- ya que invade la vida cotidiana, y abarca más y más trabajo dentro de la lógica del trabajo social abstracto. Pero dicha flexibilidad no es infinita. El trabajo diario real -de trabajo remunerado y no remunerado- no puede ser extendido sin límite.

La producción de bienes funciona en un periodo de tiempo muy corto. Como mucho, dura un ciclo de negocio (de siete a doce años). Por supuesto, los ciclos de producción son incluso más cortos, y así lo han sido en las décadas recientes, hecho que se ha manifestado en una profusión de formas "flexibles" de producción capitalista ${ }^{37}$. Como Melissa Wright nos ha mostrado ${ }^{38}$, dicha flexibilización está fundamentada en la rápida apropiación y subsecuente agotamiento de las mujeres trabajadoras a lo largo del Sur Global. La "desechable mujer trabajadora del tercer mundo" se ha convertido en un pilar del Trabajo Barato en la era neoliberal ${ }^{39}$. Ya en la década de los 70 , el $30 \%$ de las mujeres trabajadoras surcoreanas, tenían "un día de 15 horas o incluso más largo, [e] invalidez como resultado de accidentes de trabajo... incrementados por una tasa anual del $17 \% " 40$. Esto no era, por supuesto, un desarrollo original. Seccombe traza una trayectoria similar para mujeres y niños en las regiones industrializadas de Inglaterra y Francia del siglo XIX ${ }^{41}$. Lo que destacan Wright y Seccombe es el carácter históricamente transitorio de las fronteras de bienes de Trabajo Barato. En cierto punto, la flexibilidad del trabajo reproductivo no remunerado ya no es suficiente para sostener el creciente excedente ecológico.

La implicación es banal, pero soporta el énfasis tras la "gran duplicación" de la fuerza

\footnotetext{
${ }^{36}$ HILBRECHT M., et al., "I'm Home for the Kids': Contradictory Implications for Work-Life Balance of Teleworking Mothers" en Gender, Work and Organization, vol. 15, no 5, 2008, pp. 456-457.

${ }^{37}$ HAVERY, The Condition of Postmodernity, 1989.

${ }^{38}$ WRIGHT, M.W., Disposable Women and Other Myths of Global Capitalism, Routledge, New York, 2006.

${ }^{39}$ Ibíd., p. 29.

${ }^{40}$ LIPIETZ, A., "Towards Global Fordism" en New Left Review, vol. I, no 132, 1982, pp. 33-47.

${ }^{41}$ SECCOMBE, W., Weathering the Storm, Ed. Verso, London, 1995, pp. 71-80.
} 
de trabajo mundial (actual y potencial) desde 199042. La mano de obra barata no es una condición eterna de la civilización capitalista. La provisión de fuerza de trabajo y de trabajo no remunerado no es meramente una cuestión "social", pero sí una cuestión de ecologíamundo: el valor (o lo barato) de la fuerza de trabajo está directamente ligado con el trabajo no remunerado de los humanos y del resto de la naturaleza. La conexión entre la fuerza de trabajo humana y el trabajo extra-humano no es distante sino íntima, dialéctica, inmediata ${ }^{43}$

En lugar de una óptica Cartesiana -la "explotación del trabajo y la naturaleza"44_ empezaré con dos formas de trabajo-en-la-naturaleza. Uno es el trabajo remunerado dentro del sistema de bienes. El otro es el trabajo no remunerado fuera de la producción directa de bienes, pero dentro de la división del trabajo capitalista. Un método fundamentado en la doble interioridad nos permite unir esos momentos distintivos: siempre que consideramos el trabajo (trabajo-en-la- naturaleza) lo mejor es moverse inmediatamente a considerar la naturaleza-en-el- trabajo, y volver otra vez. Trabajo-en-la-naturaleza es naturaleza-en-eltrabajo. El trabajo es una coproducción del ser humano y del resto de la naturaleza; es de hecho un metabolismo, tal y como Marx indica. Y este metabolismo toma la forma de la ley de valores -como un proceso histórico conjuntivo- sustentado a través de regímenes de trabajo social abstracto y de naturaleza social abstracta, reproducidas a través de relaciones de capitalización y apropiación.

Esta relación contradictoria ha sido como quemar la vela por ambos extremos ${ }^{45}$. De un extremo encontramos el tiempo de producción del capital; del otro extremo, el tiempo de reproducción de la vida. Esta estrategia funciona mientras haya velas que quemar, y mientras producir nuevas velas sea fácil. La actividad vital de reproducir seres humanos que puedan convertirse en trabajadores es el alma del capitalismo. Si esto no ocurre de forma "barata", sino que comienza a ser más caro, la misma base del valor -fuerza de trabajo mercantilizadase vuelve un problema. Aquí la reproducción intergeneracional de la fuerza de trabajo entra en escena. Considerando la era de la Revolución Industrial, Seccombe observa como:

"El capitalismo industrial en el preciso momento de su avance triunfante revela su lado oscuro. Capitalistas privados, bajo el látigo de la competencia, mostraron una ruinosa indiferencia a la más elemental de las precondiciones de la reproducción de vida del proletariado $y$, sobre todo, de las mujeres, forzándolas a intentar reconciliar las demandas antagonistas de los ciclos diarios y generacionales de la fuerza de trabajo ${ }^{46 . "}$

¿Ha sido esto tan diferente en el largo siglo XX?

Este "látigo de la competición" existe tanto en la producción como en el mercado.

42 FREEMAN, R., "What Really Ails Europe (and America)" en The Globalist, 3 de junio de 2005.

${ }^{43}$ Aunque los críticos Verdes han enfatizado problemas con comida, energía y materias primas, el trabajo sigue siendo epifenomenal en sus análisis. Véase, por ejemplo, HEINBERG, Peak Everything, 2007; FOSTER, The Ecological Revolution, 2009; SPETH, J.G., The Bridge at the End of the World, Yale University Press, New Haven, 2008.

${ }^{44}$ FOSTER, et al., The Ecological Rift, 2010, p. 80. Énfasis añadido por el autor.

${ }^{45}$ N.d.T.: La expresión en inglés "burning the candle at both ends" aquí usada, significa trabajar o hacer cosas desde temprano por la mañana hasta tarde en la noche descansando muy poco.

${ }^{46}$ SECCOMBE, W., "Marxism and Demography" en New Left Review, vol. I, no 137, 1983, p. 44 
Impone una disciplina de tiempo en toda la producción capitalista, aunque se extiende más allá de la producción. El compromiso del capital con la productividad laboral como medida de riqueza, revela que el capitalismo es un régimen temporal: un sistema comprometido con la "aniquilación del espacio por el tiempo"47. La aniquilación del espacio, para estar seguros, pero también la aniquilación de la actividad vital por el tiempo abstracto: el impulso para obligar a toda actividad vital a trabajar bajo los ritmos del capital. La llegada de lo que Thompson denomina "tiempo industrial"48 -que precede la Revolución Industrial por varios siglos- no era simplemente un fenómeno en torno a las fábricas. Era igualmente un fenómeno familiar, y ambas transformaciones de la fábrica y la familia estaban estrechamente ligadas con el sistema de plantaciones de azúcar organizado en el tiempo industrial. En la Inglaterra del siglo XIX

"La elección de alimentos fue parcialmente calculada en términos de tiempo disponible, y no solamente en términos de costes relativos. La división laboral en el seno de la familia moldeó la evolución de las preferencias alimenticias británicas; una mujer que deja la casa para ganar un salario tuvo un efecto limitado en la dieta familiar, incluso si su trabajo incrementaba los ingresos familiares... Parece no haber duda que [el sistema de fábricas proporcionó acceso inusual] al azúcar y sus productos derivados [para trabajadores industriales, porque estas comidas permitían] el ahorro de tiempo, [y por tanto compensó parcialmente por los] trabajos agotadores que ofrecieron a mujeres y niños. El descenso del horneo de pan en la casa fue representativo del cambio de un sistema tradicional de cocina, costoso en combustible y tiempo, hacia un 'comer de conveniencia'. Conservas dulces [mermelada], que podían dejarse indefinidamente sin que se estropeasen y sin refrigeración, que eran baratas y atractivas para los niños, y que sabían mejor que muchas de las caras mantequillas con el pan comprado en tiendas, dejaron atrás o remplazaron la avena, así como el té remplazó la leche... En la práctica, las comidas de conveniencia liberaron a la esposa con salario de preparar una o incluso dos comidas por día, al mismo que tiempo que proporcionaban un gran número de calorías a toda su familia ${ }^{49}$."

Las académicas feministas frecuentemente han señalado la centralidad de la contradicción entre el tiempo de reproducción de la vida y el tiempo de reproducción del capital. Pero sus implicaciones tienen que ser todavía expandidas al capitalismo en la red de la vida. Si queremos comprender la contradicción temporal entre vida y capital como un límite de la civilización capitalista, entonces no podemos permanecer más tiempo en el binarismo Naturaleza/Sociedad. Es imposible decir que la Naturaleza externa es el límite de la civilización -por la buena razón que dichos límites son coproducidos por humanos en la naturaleza como un todo-. La naturaleza es co-producida. El capitalismo es co-producido. Los límites son coproducidos.

Si la gran preocupación de los historiadores ambientales ha sido, en este punto, el

\footnotetext{
47 MARX, Karl, Grundrisse..., op. cit., p. 524.

${ }^{48}$ THOMPSON, E.P., "Time, Work-Discipline, and Industrial Capitalism", 1967, pp. 56-97.

49 MINTZ, Sweetness and Power, 1985, p. 130
} 
espacio ${ }^{50}$, ahora es posible considerar el espacio en el tiempo ( $y$ el tiempo en el espacio). De ahí la centralidad del trabajo. Central a la ley del valor es el empuje a reducir a cero el tiempo de renovación social necesario para el capital -una ambición que está más cerca de la realidad en los actuales intercambios de alta frecuencia del siglo XXI-. Este empuje a reducir el tiempo de renovación del capital a cero es, de hecho, un momento crucial en la historia ambiental del capitalismo, llegando más allá de los dominios de la producción, intercambio, transporte, y comunicación ${ }^{51}$. El aniquilamiento del espacio por el tiempo transforma toda la vida y el espacio dentro de la fuerza gravitacional de la ley del valor. Considerando, por ejemplo, la revolución de la "cría intensiva" en producción de carne. Esta revolución efectuó la transición de los pollos de 73 días en 1955 a los pollos de 42 días en 1995 en Norte América52. Quizás incluso más dramáticamente, podemos ver esta revolución en la transformación de la producción de cerdo en China, donde un cerdo de 12 meses en 1978 se convirtió en un cerdo de 6 meses en $2011^{53}$. Aquí la "fábrica como medioambiente" está en plenitud ${ }^{54}$.

¿Es tan diferente para trabajadores humanos? El peligro es ver la "cría intensiva" como una cuestión medioambiental y la "producción de fábrica" como una cuestión social. Pero dicho dualismo simplemente esconde demasiadas cuestiones en la producción del tiempo, el espacio y la naturaleza del capitalismo. La transición del Fordismo al embalaje neoliberal de carne en los Estados Unidos - debemos recordar los orígenes decimonónicos del ensamblaje moderno en línea con el embalaje de carne americano ${ }^{55}$ - fue una transición de un trabajo bien pagado y relativamente seguro, a un trabajo mal pagado y altamente peligroso después de 1980. Esta transformación no solo dejó al embalaje de carne como el trabajo industrial más peligroso en América, sino que minó radicalmente la seguridad alimenticia, dado que comenzó a proliferar la contaminación bacterial ${ }^{56}$. Dada la centralidad de la Mano de Obra Barata, debemos también señalar la centralidad de los inmigrantes latinos en el sector del embalaje neoliberal de carne. La distribución de este Trabajo Barato fue posible por una ofensiva de clase en dos frentes. Uno tuvo lugar dentro de las fronteras nacionales, resultando en la destrucción simultánea de la pequeña burguesía agraria y del poder de la clase trabajadora industrial ${ }^{57}$-en este caso, las familias granjeras de cerdos de clase media y los trabajadores de embalaje de carne altamente organizados ${ }^{58}$-. El otro movimiento de lucha de clase asumió un carácter neocolonial y neoliberal, ya que el orden agrario mexicano fue progresivamente desestabilizado, especialmente después de 1994. El aniquilamiento del espacio por el tiempo -y sus coordenadas en la nueva coordinación de espacio-tiempo y tiempo-espacio- señaló la

50 Véase: CRONON, Nature's Metropolis, p. 1991.

${ }^{51}$ HARVEY, The Condition of Postmodernity, 1989; WARF, Time-Space Compression, 2008.

52 BOYD, W., "Making Meat" en Technology and Culture, vol. 42, no 4, 2002, pp. 631-64

${ }^{53}$ M. SCHNEIDER, Feeding China's Pigs, Institute for Agriculture and Trade Policy, Minneapolis, 2011; MACDONALD, M., y IYER, S., Skillful Means: The Challenges of China's Encounter with Factory Farming, BrighterGreen, New York, 2011.

${ }^{54}$ SELLERS, C., "Factory as Environment" en Environmental History Review, vol. 18, no 1, 1994, pp. 55-83.

${ }^{55}$ CRONON, Nature's Metropolis, 1991.

${ }^{56}$ GOUVEIA, L., y JUSKA, A., "Taming Nature, Taming Workers" en Sociologia Ruralis, vol. 42, no 4, 2002, pp. 370390.

${ }^{57}$ MOODY, K., An Injury to All, Ed. Verso, London, 1988; MACLENNAN, C., y WALKER, R., "Crisis and Change in U.S. Agriculture", en Agribusiness in the Americas, ed. R. Burbach and P. Flynn, Monthly Review Press, New York, 1980, pp. 21-40.

${ }^{58}$ Food and Water Watch, The Economic Cost of Food Monopolies, Food and Water Watch, Washington D.C., 2012; RACHLEFF, P.J., Hard-Pressed in the Heartland, South End Press, Boston, 1993. 
acumulación de capital, la búsqueda de poder, y la coproducción de la naturaleza... itodo al mismo tiempo!

Esta aceleración del cambio histórico -la comprensión espacio-temporal de la vida y el espacio ${ }^{59}$ - no es de reciente cosecha. Fue parte del crecimiento del capitalismo ${ }^{60}$. El crecimiento del "tiempo abstracto" fue central; el aniquilamiento del espacio por el tiempo pudo ocurrir sólo cuando la temporalidad pudo ser comprendida como una variable "independiente"61. ¿Independiente de qué? En primera instancia, independiente de la productividad de la tierra como base de la civilización. Cuando el poder reside en el control de la tierra, como en la Europa feudal o la China de la dinastía Song, el tiempo de la civilización era el tiempo de las estaciones, de siembra y cosecha, de nacimientos y muertes, de "cataclismos y festivales"62. Era un orden irregular del tiempo. Incluso aquí, debemos recordar que hombres y mujeres, en el poder y en la vida diaria, activamente co-producían tiempo; no eran sujetos pasivos de los ciclos "naturales". Sin embargo, la influencia de este último era fuerte, y en dichas civilizaciones, faltaba bien la capacidad o la motivación (o ambas) para crear tiempo abstracto. Estas capacidades y motivaciones comenzarían a cambiar en la Europa del siglo XIV. Los primeros relojes mecánicos aparecieron a finales del siglo XIII, y a lo largo de la crisis del largo siglo XIV, los relojes comenzaron a ser un elemento de la vida diaria en la Europa urbana e industrial. Esta transición de los relojes al tiempo del reloj fue realmente novedosa. Esto fue menos una cuestión de tecnología como de técnicas -un reloj es un reloj-. Se convirtió en tiempo del reloj, convergiendo tecnología, poder y naturaleza, solo bajo ciertas circunstancias ${ }^{63}$.

Una civilización fundamentada en el dinero y el tiempo de trabajo exigía un tipo de tiempo muy diferente. A fin de cuentas, el feudalismo europeo que permanecía en los patrones pre-modernos de auge y caída, estaba fundamentado en las dinámicas de la productividad de la tierra, la expansión de fronteras, y las relaciones señor-campesino. Pero era también una civilización sobre la base de una extraordinaria fragmentación del poder y la riqueza. Esto permitió nuevas concentraciones de proletarización y manufactura que prefiguró el auge del capitalismo, especialmente desde finales del siglo XIII. "Grandes ciudades textiles como Douai, Ypres o Bruselas... [pueden ser comparadas con] una gran fábrica", con timbres regulando el comienzo y el fin del día laboral64. A principios del siglo XIV, la hora del timbre se retiraría ante el rápido avance del tiempo-reloj; aunque aún fue un cambio menor para el tiempo abstracto, pero también fue retirado del tiempo-agrario del siglo X. A mediados del siglo XIV, "la hora uniforme de 60 minutos rápidamente... [reemplazó] los días como la unidad fundamental de tiempo de trabajo en la industria textil". El nuevo día de trabajo temporalmente segmentado comenzó a ser objeto de intensas luchas de clase durante la

\footnotetext{
59 HARVEY, The Condition of Postmodernity, 1989.

60 MOORE, "Ecology and the Rise of Capitalism" (2007); WARF, Time-Space Compression, 2008, pp. 40-77.

${ }^{61}$ POSTONE, Time, Labor, and Social Domination, 1993.

62 WHITROW, G.J., Time in History, Oxford University Press, Oxford, 1989, p. 110.

${ }^{63}$ Y así, por ejemplo, Su Sung, en la China del siglo XI, había inventado un reloj mecánico, pero uno diseñado para el Emperador, no para la vida diaria. NEEDHAM, J., et al., Heavenly Clockwork: The Great Astronomical Clocks of Medieval China, 2nd ed. Cambridge University Press, Cambridge, 1986 [1960 orig.].

${ }^{64}$ CARUS-WILSON, E.M., "The Woolens Industry", en The Cambridge Economic History of Europe, Vol. 2, eds. M. Postan y E.E. Rich, Cambridge University Press, Cambridge, 1952, p. 644.
} 
prolongada crisis feudal65. De hecho, es en esta era de crisis de época que encontramos los orígenes de la idea de productividad laboral con su sensibilidad que "el tiempo es dinero"66.

Hacia finales del siglo XIV, el tiempo-reloj, con sus horas de 60 minutos, "fue firmemente establecido en las grandes áreas urbanizadas de Europa Occidental, reemplazando el día como la unidad básica de tiempo"67. Y si los primeros movimientos de tiempo abstracto se habían originado en los monasterios, para 1370 -al menos en Francia- el tiempo-reloj estaba implacablemente secularizado: "el nuevo tiempo... [se había convertido] en el tiempo del estado"68. Lo que distinguió este nuevo tiempo no era simplemente su linealidad y regularidad, sino las formas en las que el tiempo fue representado como "exterior a la vida"69. El reloj (como técnica) hizo por el tiempo lo que la perspectiva Renacentista hizo por el espacio. Se "disoció el tiempo de los eventos humanos y... creó la creencia en un mundo independiente de las secuencias matemáticas medibles"70.

A la llegada del siglo XVI, el impulso exterior de los capitalistas y estados europeos fundía el tiempo-reloj con el "tiempo del comerciante" en su sentido más amplio71. Empezamos a ver nuevas formas de tiempo-mundo -tiempo abstracto- que eran más que "meros medios de dar seguimiento a las horas". El tiempo abstracto comenzó a significar "sincronizar las acciones del hombre" y la naturaleza ${ }^{72}$, en un nuevo tapiz de dinero, producción de bienes, y poder estatal.

Esta "revolución en tiempo"73 subyace en la temprana revolución capitalista en el espacio, y moldea la aceleración del cambio de paisaje que ocurrió tras 1450 (ver Capítulo Siete $\left.^{74}\right)$. Esta aceleración está arraigada en la histórica relación del valor como un proyecto utópico, el cual tiene una correspondencia con el mundo real en la aceleración del cambio medioambiental: de ahí la importancia del proyecto de correspondencia del capital. Este proyecto, como hemos visto, busca reducir el tiempo de vida al tiempo de acumulación. Esto es obviamente imposible. Sin embargo, el deseo del capitalismo instantáneo anima las crudas compulsiones de la acumulación mundial. Es, por tanto, no solo la "radical simplificación" de paisajes y otras naturalezas que revelan la ley del valor en el trabajo; es también el impulso de hacer el "tiempo de la naturaleza" aún más cercano al "tiempo del capital".

Este es el proyecto de correspondencia del capitalismo, a través del cual el capital busca rehacer la realidad a su propia imagen y de acuerdo a sus propios ritmos. Los paisajes

\footnotetext{
${ }^{65}$ WHITROW, G.J., Time in History, Oxford University Press, Oxford, 1989, p. 108; LE GOFF, J., Time, Work and Culture in the Middle Ages, University of Chicago Press, Chicago, 1980, pp. 43-52.

${ }^{66}$ LANDES, D., The Wealth and Poverty of Nations, W.W. Norton, New York, 1998, pp. 49-50.

${ }^{67}$ LE GOFF, J., Time, Work and..., op. cit., p. 49.

${ }^{68}$ Ibíd., p. 50.

${ }^{69}$ GUREVICH, A.J., "Time as a Problem of Cultural History", en Cultures and Time, ed. L. Gardet et al., UNESCO Press, Paris, 1976, p. 241.

70 MUMFORD, Technics and Civilization (1934), p. 15.

${ }^{71}$ LE GOFF, Time, Work and..., op. cit.

72 MUMFORD, Technics and..., op. cit., p. 14.

73 LANDES, Revolution in Time (1983).

${ }^{74}$ N.d.T.: Capítulo séptimo del libro MOORE, Jason, Capitalism in the Web of Life, Editorial Verso, Londres, 2015.
} 
agrícolas se agotan porque el capital debe extraer trabajo no remunerado más rápido que lo que las relaciones agro-ecológicas se pueden auto-reproducir. Las clases trabajadoras se agotan porque el capital debe extraer el excedente del trabajo tan rápido como sea posible. Los capitalistas particulares ganan en el proceso, pero con el tiempo, el capitalismo como un todo sufre por el rápido proceso de capitalización de los costes de reproducción de todo el sistema. La parte de trabajadores no remunerados desciende. El excedente ecológico cae.

\section{El auge y caída de la Naturaleza Barata: el Momento Neoliberal}

¿Puede observarse la tendencia a la caída del excedente ecológico durante la era neoliberal? Debemos recordar que un alto excedente ecológico mundial representa una ratio de baja capitalización a alta apropiación. Esta es una condición necesaria para el revivir de la acumulación. El "boom" neoliberal que comenzó después de 1983 estuvo acompañado -o precedido- por un significante descenso cíclico de los precios de la comida, energía y recursos. Los precios de los bienes para la comida descendieron un 39\% -y los metales a la mitadentre 1975 y 1989. Mientras tanto, el petróleo se estabilizó hacia 1983 para los siguientes veinte años, a un precio por barril cerca del doble que el de la era de posguerra75.

Pero no fue solo las naturalezas extra-humanas que se abarataron.

El revivir de la acumulación de la década de los 80 también se convirtió en Trabajo Barato. Esto supuso un régimen de acumulación que podía suministrar tanto trabajo remunerado como sin remunerar en volumen suficiente como para restaurar la acumulación. En términos formales, restablecer el Trabajo Barato significó reducir el valor de la fuerza de trabajo. Esto no fue fácil de llevar a cabo. Había cinco dimensiones clave del proyecto neoliberal para restaurar el Trabajo Barato después de 1973. La primera fue represión salarial. Burgueses alrededor del Norte Global comenzaron a organizarse como clase, y moverse agresivamente contra los sindicatos tras la recesión de 1974-197576. La represión salarial fue especialmente importante ya que el crecimiento de la productividad laboral se hundió en la década de los 70, una desaceleración que cada vez parecía más permanente ${ }^{77}$. Segundo, la caída de la tasa de beneficio en la industria americana -inducida tanto por el poder de la clase trabajadora como por el crecimiento de la composición orgánica del capital- permitió a capitalistas americanos y otros moverse rápidamente hacia la "fábrica global" en la década de los $70^{78}$. Esto fue un cambio tectónico en la historia mundial que implicó la simultánea des-industrialización de zonas centrales y la rápida industrialización del Sur Global ${ }^{79}$. Tercero,

75 MCMICHAEL, P., "Global Development and the Corporate Food Regime" en New Directions in the Sociology of Global Development, ed. HTEL, F. y MCMICHAEL, P., Elsevier, Oxford, 2005; RADETZKI, M., "The Anatomy of Three Commodity Booms" en Resources Policy, no 31, 2006, pp. 56-64; VAN DER MENSBRUGGHE, D., et al., "Macroeconomic Environment and Commodity Markets", en Looking Ahead in World Food and Agricultre, ed. P. Conforti FAO, Rome, 2011; MGI [McKinsey Global Institute], "MGI's Commodity Price Index - an Interactive Tool", (2014), www.mckinsey.com

76 MOODY, An Injury to All, 1988.

77 GORDON, R.J., Revisiting U.S. Productivity Growth over the Past Century with a View of the Future, National Bureau of Economics Research, Cambridge, 2010 Working Paper, 15834; GORDON, R.J., "Is U.S. Economic Growth Over?", 2012.

${ }^{78}$ BARNET, R., The Lean Years (New Year: Simon and Schuster, 1980); GORDON, D.M., et al., Segmented Work, Divided Workers, Cambridge University Press, Cambridge, 1982.

${ }^{79}$ ARRIGHI, G., et al., "Industrial Convergence, Globalization, and the Persistence of the North-South Divide" en Studies in Comparative International Development, vol. 38, no 1, 2003, pp. 3-31. 
la fábrica global dependió del "gran recinto global" que comenzó a principios de la década de los $80^{80}$. Estos recintos globales, realizados a través de programas de ajuste estructural y liberalización del mercado, reestructuraron las relaciones de clase agrarias a lo largo del mundo, desposeyendo a centenas de millones de agricultores en todo el mundo. Sólo en China, entre 200-300 millones de migrantes se movieron del campo a la ciudad ${ }^{81}$. Este nuevo proletariado global empequeñeció cualquier otro que hubiera venido antes. En concierto con la apertura de Rusia, China e India al mercado global, el proletariado global se dobló tras 198982. Cuarto, esta "gran duplicación" representó una aún mayor expansión del proletariado femenino, agregando el trabajo remunerado por encima del trabajo no remunerado en una escala sin precedentes. La proletarización neoliberal fue, en este ajuste, una expansión global sin precedentes del "segundo cambio" de Hochschild83. Finalmente -y prácticamente ignorado universalmente por los medioambientalistas- el Trabajo Barato se hizo posible a través de un nuevo régimen de "subconsumo forzado", manifestado en la represión salarial en el Norte y el descenso del bienestar a lo largo del Sur (exceptuando China) ${ }^{84}$.

En el 2003, el excedente ecológico mundial paró de crecer y comenzó a decrecer. Registrado por el boom de bienes, primero de lento movimiento y más tarde rápido, esta fue la señal de la crisis del neoliberalismo como una forma de organizar la naturaleza. Esta expresión de señales de crisis comenzó con una contracción cíclica del excedente ecológico; su más claro indicador fue el aumento del precio de los bienes de metales, energía y alimento. Pero esto no fue solo un boom de bienes, entre otras cosas por su inusual duración -aunque pasado su pico en términos de precios (al menos por ahora), queda un "boom" en el sentido que los precios aún están considerablemente por encima de las medias entre 1980-2000-. ¿Qué nos indica este boom de bienes que parece sin fin? Como mínimo, el peculiar carácter de este boom -que incluye más bienes primarios, dura más, y donde se vio una mayor volatilidad de los precios que en ningún otro boom de bienes en la historia moderna universal ${ }^{85}$ indica un agotamiento de la estrategia neoliberal de la Naturaleza Barata. Notablemente, las estrategias neoliberales para reducir los precios de los Cuatro Grandes productos comenzaron a fallar al menos cinco años antes de los eventos financieros del 2008. Los economistas hablan de este largo boom de bienes como un "superciclo" - una larga década de incremento de precios en los bienes básicos-. Pero hasta ahora ellos han invocado un "mundo de escasez" abstracto ${ }^{86}$, más que considerar la posibilidad de que el superciclo de hoy representa un límite histórico al régimen capitalista de larga duración de la Naturaleza Barata.

La erosión del Trabajo Barato es una insinuación de dichos límites co-producidos. En otras palabras, la señal de crisis del neoliberalismo no es una mera cuestión de naturalezas extrahumanas -reflejadas en el boom de bienes- sino de la naturaleza humana también. En

\footnotetext{
${ }^{80}$ ARAGHI, F., "The Great Global Enclosure of Our Times", 2000.

81 WEBBER, M., "The Dynamics of Primitive Accumulation" en Environment and Planning, A44, no 3, 2012, pp. 560579.

${ }^{82}$ FREEMAN, "What Really Ails Europe (and America)" 2005.

${ }^{83}$ HOCHSCHILD, The Second Shift, 1989.

${ }^{84}$ ARAGHI, "Accumulation by Displacement" 2009.

85 WORLD BANK, Global Economic Prospects 2009, 2009.

${ }^{86}$ JACKS, "From Boom to Bust?", 2013.
} 
China, los salarios reales se incrementaron un $300 \%$ entre 1990 y $2005^{87}$. Los salarios en el sector de manufacturas crecieron seis veces más rápido que la tasa de inflación, y los costes unitarios del trabajo se incrementaron un $85 \%$ entre 2000 y $2011^{88}$. El crecimiento de los costes unitarios del trabajo es el más peculiar de todos dado el espectacular incremento de la productividad laboral: el rendimiento por trabajador chino creció un 7.2\% anual entre 1993 y $2013^{89}$.

Mientras tanto, la usual estrategia de mover las fronteras del Trabajo Barato -buscando nuevos grupos de trabajo no remunerado en apoyo de los trabajadores de bajos salarios- está en movimiento, pero con rendimientos decrecientes. En China, la política gubernamental de "Ir hacia el Oeste" que tenía como objetivo atraer la industria al interior, ha estrechado los costes laborales entre el interior y las regiones costeras a una "sorprendente... diferencial salarial insignificante"90. La migración del campo a la ciudad se ha ralentizado considerablemente en los últimos años ${ }^{91}$. En el 2012, la inversión extranjera por cápita en Camboya adelantó a China ${ }^{92}$. Pero Camboya es mucho más pequeña que China, que es parte del problema más amplio: las fronteras se están contrayendo en el preciso instante en el que el capital necesita las mayores fronteras de bienes de siempre para resolver el problema de sobreacumulación. Mientras tanto, las tecnologías de la información y de la comunicación que hicieron posible la producción global son ahora usadas en la lucha de clases:

"Hoy los trabajadores en Camboya han comenzado la acción sindical solo desde hace unos años, no desde hace veinticinco. Hay huelgas y presiones por mayores salarios y beneficios que los que están recibiendo. Esto por supuesto reduce el valor para que las multinacionales se muevan a Camboya, o Myanmar, o Vietnam, o Filipinas. Ahora resulta que lo que se ahorraban en moverse de China no era tanto93."

La erosión del Trabajo Barato que está en marcha no es exclusiva de la historia en el Este asiático. Menos comprendida, pero no menos significante, es la transición a lo largo del Norte Global hacia un "segundo (y tercer) cambio" -trabajo asalariado más el trabajo reproductivo no remunerado-. Esta transición promulgó y encarnó una de las últimas grandes fronteras de bienes del capitalismo histórico. El trabajo doméstico no remunerado ha sido el pilar de la mercantilización sin fin desde el siglo XVI ${ }^{94}$. En el Norte Global, y especialmente en Norte América, tras 1970 hemos asistido a la aceleración de la proletarización de las mujeres. Esto marca la muerte de la familia Fordista de un solo ingreso y el crecimiento del hogar "flexible" con doble ingreso. Esta aceleración de la década de los 70 ha sido prefigurada por

87 Midnightnotes.org, "Promissory Notes. From Crisis to Commons" (2009).

88 USDC [United States Department of Commerce], "Assess Costs Everywhere", 2013, [Consultado el 24 de abril del 2013]: acetool.commerce.gov/labor-costs.

${ }^{89}$ ILO [International Labour Office], Global Employment Trends 2014: Risk of a Jobless Recovery?, International Labour Office, Geneva, 2014, p. 52.

${ }^{90}$ SCOTT, J., "Who Will Take Over China's Role as the World's Factory Floor?" en Saturna Sextant Newsletter, August 2011, p. 1.

${ }^{91}$ FEGLEY, B., "30 Years of Double-Digit Chinese Growth" en From the Yardarm, vol. 7, n 1, 2013.

92 BRADSHER, K., "Wary of China, Companies Head to Cambodia" en New York Times, 8 de Abril de 2013.

${ }^{93}$ WALLERSTEIN, I., "End of the Road for Runaway Factories?" Commentary 351 (15 de abril de 2013). [Consultado el 14 de noviembre de 2013]: www2.binghamton.edu/fbc/commentaries/archive-2013/351en.htm

${ }^{94}$ MIES, M., Patriarchy and Accumulation, 1986 
el desarrollismo soviético ${ }^{95}$, y también por la rápida entrada de las mujeres americanas al trabajo remunerado desde la década de los $30^{96}$. Estas, también fueron fronteras de bienes, marcadas por la progresiva mercantilización del potencial de trabajo y de la progresiva apropiación de los "regalos gratis" de la naturaleza (humana). De ahí la imposición de múltiples "cambios", y de la doble opresión en el tiempo de las mujeres vía las presiones simultáneas de capitalización y apropiación; incluso tan pronto como a mitad de década de los 60 un creciente número de mujeres casadas americanas cambiaron sus trabajos de 55 horas semanales en casa por 76 horas semanales de trabajo en casa (y pagado) ${ }^{97}$. Si esto fuera todo -como en la interpretación de Hochschild de la frontera de bienes ${ }^{98}$ - habría poco que añadir. Lo que la teoría de las fronteras de bienes ilumina no es solo el patrón de sucesivos movimientos emparejados de mercantilización/apropiación, sino las oportunidades finitas inscritas en cada uno de dichos movimientos. En los Estados Unidos, el extraordinariamente rápido incremento de participación de las madres en la fuerza de trabajo $-50 \%$ entre 1975 y $1995^{99}$ - no fue solo un momento poderoso de la represión salarial neoliberal mientras mantenían la demanda efectiva (del consumidor); fue también un negocio único. La frontera de bienes es un ticket de ida. Las fronteras, una vez apropiadas y mercantilizadas, no son fronteras nunca más. Estas, sin embargo, han avanzado como hemos visto en el despliegue de la relación proletaria para las mujeres a lo largo del Sur Global desde la década de los $80^{100}$.

\section{Conclusión}

La apropiación del trabajo doméstico no remunerado y de la generosidad de la naturaleza extrahumana -ambos trabajo no remunerado- no es un residuo de la producción real en el capitalismo. Más que cíclicas e implacables expansiones de la zona de apropiación del trabajo no remunerado son, junto con la revolucionada producción de bienes, el requerimiento decisivo para la acumulación. El imperativo que enfrenta el capital para expandir la zona de trabajo no remunerado más rápido que la capitalización del oikeios, es la base histórica a través de la cual el poder capitalista agrupó los créditos de la época de "mujeres, naturaleza, y colonias"101. Sin las mujeres, naturaleza y colonias - una lista estilizada para estar segurosla acumulación falla. La apropiación del trabajo devaluado debe necesariamente tener más peso que la capitalización del trabajo a menos que los Cuatro Grandes insumos (fuerza de trabajo, comida, energía y materias primas) comiencen a aumentar, y las oportunidades de acumulación a través de la producción e intercambio de bienes ( $\left.M-C-M^{\prime}\right)$ comiencen a descender.

95 SACKS, M., "Unchanging Times" en Journal of Marriage and Family, vol. 39, no 4, 1977, pp. 793-805.

${ }^{96}$ GOLDIN, C., "Gender Gap", en The Concise Encyclopedia of Economics, ed. D.R. Henderson, 2008. [Consultado el 1 de mayo de 2013]: www.econlib.org/library/Enc/GenderGap.html.

97 HARTMANN, H.I., "The Family as the Locus of Gender, Class, and Political Struggle" en Signs, vol. 6, nº 3, 1981, pp. 366-394.

${ }^{98}$ HOCHSCHILD, A., "The Commodity Frontier", Center for Working Families, University of California, Berkeley, 2002, Working Paper No 1.

${ }_{99}$ BLS (Bureau of Labor Statistics), "Labor Force Participation Rate of Mothers, 1975-2007", The Editor's Desk, (8 de enero de 2009). [Consutlado el 1 de mayo de 2013]: www.bls.gov/opub/ted/2009/jan/wkl/art04.htm.

100 KABEER, N., Marriage, Motherhood and Masculinity in the Global Economy, Institute for Development Studies, University of Sussex, 2007, IDS Working Paper 290; MCMICHAEL, Development and Social Change, 5th ed., 2012.

101 MIES, M., Patriarchy and Accumulation, 1986. 
La posibilidad del "final" del Trabajo Barato, solo puede ser adecuadamente entendida a través de los nexos centrales sistémicos de la división capitalista del trabajo: la relación entre alimento y fuerza de trabajo. A esto nos dirigimos ahora ${ }^{102}$. Editorial Verso, Londres, 2015. 


\section{RELACIONES INTERNACIONALES}

Revista académica cuatrimestral de publicación electrónica Grupo de Estudios de Relaciones Internacionales (GERI) Universidad Autónoma de Madrid, España

www.relacionesinternacionales.info

ISSN 1699 - 3950

ff facebook.com/RelacionesInternacionales

twitter.com/RRInternacional 